TRANSACTIONS OF THE

AMERICAN MATHEMATICAL SOCIETY

Volume 348, Number 6, June 1996

\title{
AN APPLICATION OF CONVEX INTEGRATION TO CONTACT GEOMETRY
}

\author{
HANSJÖRG GEIGES AND JESÚS GONZALO
}

\begin{abstract}
We prove that every closed, orientable 3-manifold $M$ admits a parallelization by the Reeb vector fields of a triple of contact forms with equal volume form. Our proof is based on Gromov's convex integration technique and the $h$-principle. Similar methods can be used to show that $M$ admits a parallelization by contact forms with everywhere linearly independent Reeb vector fields. We also prove a generalization of this latter result to higher dimensions. If $M$ is a closed $(2 n+1)$-manifold with contact form $\omega$ whose contact distribution $\operatorname{ker} \omega$ admits $k$ everywhere linearly independent sections, then $M$ admits $k+1$ linearly independent contact forms with linearly independent Reeb vector fields.
\end{abstract}

\section{INTRODUCTION}

A contact form on a smooth 3-manifold $M$ is a differential 1-form $\omega$ such that $\omega \wedge d \omega$ is nowhere zero (i.e., a volume form). Given a contact form $\omega$, there is a unique vector field $\xi$ such that $\omega(\xi)=1$ and $\xi\rfloor d \omega=0$ (Here '」' denotes the interior product.) This vector field is called the Reeb vector field of $\omega$. On the contact distribution $\operatorname{ker} \omega$ one has a conformal symplectic structure defined by $d \omega$, and a compatible linear endomorphism $\phi$ satisfying $\phi^{2}=-1$. This can be extended to an endomorphism of the tangent bundle $T M$ by setting $\phi(\xi)=0$. The pair $(\phi, \xi)$ defines a reduction of the structure group of $T M$ to $U(1) \times 1$.

In [2], one of us showed that every closed, orientable 3-manifold admits a parallelization by contact forms, i.e., a triple of contact forms $\omega_{1}, \omega_{2}, \omega_{3}$ such that $\omega_{1} \wedge \omega_{2} \wedge \omega_{3}$ is nowhere zero. In the present note, we prove the following related result.

Theorem 1. Every closed, orientable 3-manifold $M$ admits a triple of contact forms $\omega_{1}, \omega_{2}, \omega_{3}$ such that the Reeb vector fields $\xi_{1}, \xi_{2}, \xi_{3}$ trivialize the tangent bundle TM. Furthermore, $\omega_{1}, \omega_{2}, \omega_{3}$ may be assumed to have the same volume form $\omega_{i} \wedge d \omega_{i}$.

One can then define a Riemannian metric $g$ on $M$ by setting

$$
g\left(\xi_{i}, \xi_{j}\right)=0 \quad \text { for } i \neq j
$$

and

Note that

$$
g\left(\xi_{i}, \xi_{i}\right)=d \omega_{1}\left(\xi_{2}, \xi_{3}\right)
$$

$$
\omega_{1} \wedge d \omega_{1}=\omega_{2} \wedge d \omega_{2}=\omega_{3} \wedge d \omega_{3}
$$

Received by the editors December 8, 1992.

1991 Mathematics Subject Classification. Primary 53C15, 53C23.

(C)1996 American Mathematical Society 
implies

$$
d \omega_{1}\left(\xi_{2}, \xi_{3}\right)=d \omega_{2}\left(\xi_{3}, \xi_{1}\right)=d \omega_{3}\left(\xi_{1}, \xi_{2}\right) \neq 0
$$

hence

$$
g\left(\xi_{1}, \xi_{1}\right)=g\left(\xi_{2}, \xi_{2}\right)=g\left(\xi_{3}, \xi_{3}\right) \neq 0 .
$$

If we define linear endomorphisms $\phi_{1}, \phi_{2}, \phi_{3}$ of $T M$ by

$$
\phi_{1} \xi_{1}=0, \quad \phi_{1} \xi_{2}=\xi_{3}, \quad \phi_{1} \xi_{3}=-\xi_{2}
$$

(and cyclic permutations thereof), then the $\phi_{i}$ satisfy the quaternionic identities

$$
\begin{aligned}
& \phi_{i}^{2}=-1 \quad \text { on }\left\langle\xi_{i}\right\rangle^{\perp}, \\
& \phi_{i} \phi_{j}=\phi_{k}+\xi_{i} \otimes \eta_{j}
\end{aligned}
$$

for any cyclic permutation $\{i, j, k\}$ of $\{1,2,3\}$, where $\eta_{1}, \eta_{2}, \eta_{3}$ is the coframe dual to $\xi_{1}, \xi_{2}, \xi_{3}$.

Furthermore, we have

$$
g\left(\phi_{i} X, Y\right)=d \omega_{i}(X, Y), \quad i=1,2,3
$$

for all vector fields $X, Y$ on $M$. Such a family of tensors $\left(\omega_{i}, \phi_{i}, \xi_{i}, \eta_{i}\right), i=1,2,3$, together with a Riemannian metric $g$, satisfying equations (1), (2), (3), (4), is a hypercontact structure in dimension 3, as defined in [1]. This type of structure is actually defined in any dimension $4 n+3$ by the same family of tensors (the index $i$ still runs from 1 to 3 ), but one has to add to equations (1)-(4) above the duality equations

$$
\eta_{i}\left(\xi_{j}\right)=\delta_{i j},
$$

which in dimension 3 are true by definition of the dual coframe, and the relations

$$
g\left(\phi_{i} X, \phi_{i} Y\right)=g(X, Y) \quad \text { for } X, Y \in \operatorname{ker} \eta_{i},
$$

which hold automatically in dimension 3 . See Remark 2 below for the motivation of this notion.

We shall also prove that one can obtain a simultaneous parallelization of $M$ by contact forms and Reeb vector fields.

Theorem 2. Every closed, orientable 3-manifold $M$ admits a triple of everywhere linearly independent contact forms $\omega_{1}, \omega_{2}, \omega_{3}$ with everywhere linearly independent Reeb vector fields $\xi_{1}, \xi_{2}, \xi_{3}$.

Our proof of Theorems 1 and 2 is based on the $h$-principle and the convex integration technique of Gromov [3]. In order to make this paper reasonably selfcontained, we shall give a brief outline of Gromov's theory in Section 3.

Remarks. Theorems 1 and 2 are proved by slightly different methods, and we have not been able to prove Theorem 2 under the condition that the volume forms $\omega_{i} \wedge d \omega_{i}$ be equal. However, observe that the structure equations of a hypercontact structure can be satisfied without the condition that the $\omega_{i}$ have equal volume form if we replace the Reeb vector fields $\xi_{i}$ by suitable multiples $\tilde{\xi}_{i}=f_{i} \xi_{i}$, where the $f_{i}$ are nowhere zero functions on $M$.

(2) Given a hyperkähler manifold $\left(N^{4 n+4}, g, \Omega_{1}, \Omega_{2}, \Omega_{3}\right)$ and a vector field $X$ that is a Liouville vector field with respect to all three Kähler forms $\Omega_{i}$, that is,

$$
\left.L_{X} \Omega_{i}=d(X\rfloor \Omega_{i}\right)=-\Omega_{i}, \quad i=1,2,3,
$$


it is an easy check that the forms $\left.\omega_{i}=X\right\rfloor \Omega_{i}$ define a hypercontact structure on any hypersurface transverse to $X$. (For the $\xi_{i}$ take the Reeb vector fields of the $\omega_{i}$, set $\eta_{i}=\omega_{i}$, and define the $\phi_{i}$ by (1) and on the common kernel of the $\omega_{i}$ as the restrictions of the complex structures $J_{i}$ on $N^{4 n+4}$.) This yields the basic examples $\mathbb{R}^{4 n+3}$ and $S^{4 n+3}$ as hypersurfaces in $\mathbb{H}^{n+1}$.

This construction is extended in [1] to show that under certain conditions it is possible to perform 0 -surgery on hypercontact manifolds. In particular, this gives rise to the examples $\#_{k}\left(S^{1} \times S^{4 n+2}\right)$.

The methods used in the present paper are not strong enough to construct hypercontact structures in dimension greater than 3, but Theorem 7 below may be seen as a first step in this direction, since a triple of contact forms with everywhere linearly independent Reeb vector fields is a necessary condition for a hypercontact structure. For example, the conditions of Theorem 7 (with $k=2$ ) are met by all 2-connected 7-manifolds, see [1].

\section{ACKNOWLEDGEMENTS}

This research was carried out while the second author was visiting Stanford University. He takes this opportunity to thank Yakov Eliashberg for his kind invitation and the Stanford Department of Mathematics for its support.

We also wish to thank Yakov Eliashberg for useful conversations on the $h$ principle.

\section{Reduction of the Problem}

As in [2], we use the branched cover theorem of Hilden, Montesinos, and Thickstun [4].

Theorem 3. Let $M$ be a closed, orientable 3-manifold. There exists a simple 3fold branched cover $p: M \rightarrow S^{3}$ such that the set $C$ of points in $M$ where $p$ is not a local diffeomorphism bounds an embedded disc in $M$. In particular, there is an embedded 3-ball $B$ in $M$ with $C \subset \stackrel{\circ}{B}$.

Given such a branched cover, it was shown in [2] that one can construct a contact form $\omega_{1}$ on $M$ which outside $B$ is just the lift of the standard contact form

$$
\alpha_{1}=x_{1} d x_{2}-x_{2} d x_{1}+x_{3} d x_{4}-x_{4} d x_{3}
$$

on $S^{3} \subset \mathbb{R}^{4}$, and such that the 2-plane bundle $\operatorname{ker} \omega_{1}$ on $M$ is trivial. This implies the existence of 1 -forms $\alpha$ and $\beta$ on $M$ such that $\omega_{1} \wedge \alpha \wedge \beta$ is nowhere zero, and hence $\omega_{1}, \omega_{1}+\epsilon \alpha$ and $\omega_{1}+\epsilon \beta$ give a parallelization of $M$ by contact forms if the constant $\epsilon>0$ is chosen sufficiently small.

Observe that a trivialization of $T M$ by Reeb vector fields $\xi_{1}, \xi_{2}, \xi_{3}$ corresponding to contact forms $\omega_{1}, \omega_{2}, \omega_{3}$ is equivalent to a trivialization of $\Lambda^{2} T^{*} M$ by $d \omega_{1}, d \omega_{2}$, $d \omega_{3}$. We now reduce Theorem 1 to the following proposition.

Proposition 4. Given a closed, orientable 3-manifold $M$, there are 1-forms $\alpha, \tilde{\alpha}$ and a contact form $\omega$ such that

$$
d \omega, L_{\xi} d \alpha, L_{\xi} d \tilde{\alpha}
$$

trivialize $\Lambda^{2} T^{*} M$, where ' $L$ ' denotes the Lie derivative and $\xi$ is the Reeb vector field of $\omega$. 
Proof of Theorem 1. Given $\omega, \alpha, \tilde{\alpha}$ as in Proposition 4, define vector fields $X$ and $\widetilde{X}$ on $M$ by

$$
X\rfloor(\omega \wedge d \omega)=d \alpha, \quad \tilde{X}\rfloor(\omega \wedge d \omega)=d \tilde{\alpha} .
$$

These defining equations imply that $X$ and $\widetilde{X}$ have zero divergence with respect to the volume form $\omega \wedge d \omega$, i.e.,

$$
L_{X}(\omega \wedge d \omega)=L_{\widetilde{X}}(\omega \wedge d \omega)=0 .
$$

Hence, if we denote by $\varphi_{t}$ (resp. $\tilde{\varphi}_{t}$ ) the flow corresponding to $X$ (resp. $\widetilde{X}$ ), the contact forms

and

$$
\omega_{t}=\varphi_{t}^{*} \omega
$$

$$
\tilde{\omega}_{t}=\tilde{\varphi}_{t}^{*} \omega
$$

have the same volume form as $\omega$.

We now claim that $\omega_{1}=\omega, \omega_{2}=\omega_{t}$ and $\omega_{3}=\tilde{\omega}_{t}$ are the desired contact forms for small enough $t$, i.e., we want to show that $d \omega_{1}, d \omega_{2}, d \omega_{3}$ define a trivialization of $\Lambda^{2} T^{*} M$.

It is clear that $\xi$ is also divergence-free with respect to $\omega \wedge d \omega$. Since the Lie derivative commutes with contraction, we obtain

$$
\begin{aligned}
L_{\xi} d \alpha & \left.\left.=L_{\xi}(X\rfloor(\omega \wedge d \omega)\right)=\left(L_{\xi} X\right)\right\rfloor(\omega \wedge d \omega) \\
& =[\xi, X]\rfloor(\omega \wedge d \omega)=-[X, \xi]\rfloor(\omega \wedge d \omega) \\
& \left.\left.=-\left(L_{X} \xi\right)\right\rfloor(\omega \wedge d \omega)=-L_{X}(\xi\rfloor(\omega \wedge d \omega)\right) \\
& =-L_{X} d \omega .
\end{aligned}
$$

Similarly, we have $L_{\xi} d \tilde{\alpha}=-L_{\widetilde{X}} d \omega$. Now

$$
\begin{aligned}
d \omega_{t} & =d \omega+t L_{X} d \omega+O\left(t^{2}\right) \\
& =d \omega-t L_{\xi} d \alpha+O\left(t^{2}\right)
\end{aligned}
$$

and

$$
d \tilde{\omega}_{t}=d \omega-t L_{\xi} d \tilde{\alpha}+O\left(t^{2}\right) .
$$

So for small enough $t>0$, the 2 -forms $d \omega, d \omega_{t}$ and $d \tilde{\omega}_{t}$ are everywhere linearly independent. This completes the proof of Theorem 1.

\section{Convex Integration And the $h$-Principle}

Our proof of Proposition 4 is based on the $C^{\perp}$-dense $h$-principle [3, p. 172], which is proved by the convex integration technique. For the convenience of the reader and to fix our notation, we give a brief description of the general $h$-principle and state the $C^{\perp}$-dense $h$-principle in the form in which we shall apply it in the next section.

The general setup in [3] is the following. Let $p: X \rightarrow V$ be a smooth fibration and let $X^{(r)}$ be the space of $r$-jets of germs of smooth sections $f: V \rightarrow X$. The $r$-jet of a $C^{r}$-section $f$ is denoted by $J_{f}^{r}: V \rightarrow X^{(r)}$. A section $\varphi: V \rightarrow X^{(r)}$ is called holonomic if $\varphi=J_{f}^{r}$ for some $f: V \rightarrow X$.

A differential relation is a subset $\mathcal{R} \subset X^{(r)}$. One says that $\mathcal{R}$ satisfies the $h$ principle if every continuous section $V \rightarrow \mathcal{R}$ is homotopic to a holonomic section $V \rightarrow \mathcal{R}$ by a continuous homotopy of sections $V \rightarrow \mathcal{R}$. The $h$-principle is called $C^{0}-$ dense if for any section $\psi: V \rightarrow \mathcal{R}$ such a homotopy exists in an arbitrarily small neighbourhood of $\psi(V)$. One says that the $h$-principle for extensions is satisfied 
if, given closed subsets $C_{0} \subset C \subset V$, and a section of $\mathcal{R}$ in a neighbourhood of $C$ which is holonomic in a neighbourhood $U_{0}$ of $C_{0}$, there is a homotopy to a holonomic section of $\mathcal{R}$ over $C$ such that the homotopy is constant in a neighbourhood $U_{0}^{\prime} \subset U_{0}$ of $C_{0}$.

Now suppose that $V$ is a smooth manifold that splits as $V=V^{\prime} \times[0,1]$. Furthermore, assume that the fibration $p: X \rightarrow V$ is trivial with fibre $\mathbb{R}^{q}$, so that sections of $X \rightarrow V$ can be identified with maps $f: V \rightarrow \mathbb{R}^{q}$. Fix local coordinates $u_{1}, \ldots, u_{n-1}$ in $V^{\prime}$ and let $t$ be the coordinate in $[0,1]$. Then $J_{f}^{r}$ is given by the totality of partial derivatives of order $\leq r$ with respect to $u_{1}, \ldots, u_{n-1}, t$. Define $J_{f}^{\perp}$ by $J_{f}^{r}=J_{f}^{\perp} \oplus \frac{\partial^{r} f}{\partial t^{r}}$. By $C^{\perp}$-convergence of maps we mean $C^{0}$-convergence of the jets $J^{\perp}$.

Since a jet is completely determined by a germ of a section, the concept of $C^{\perp}$-convergence easily generalizes to arbitrary fibre bundles $\mathbb{R}^{q} \hookrightarrow X \stackrel{p}{\rightarrow} V$ over a manifold $V$ as follows. Let $\tau$ be a hyperplane distribution in the tangent bundle $T V$. For a section $f: V \rightarrow X$, one can define $J_{f}^{\perp}$ in such a way that

$$
J_{f_{1}}^{\perp}=J_{f_{2}}^{\perp} \text { if and only if } D J_{f_{1}}^{r-1}\left|\tau=D J_{f_{2}}^{r-1}\right| \tau,
$$

where $D J_{f_{i}}^{r-1}$ is the differential $T V \rightarrow T\left(X^{(r-1)}\right)$.

Let $X_{\tau}^{\perp}$ be the space of $J^{\perp}$-jets. Then there are natural affine bundle projections

$$
X^{(r)} \stackrel{p_{\perp}^{r}}{\longrightarrow} X_{\tau}^{\perp} \stackrel{p_{r-1}^{\perp}}{\longrightarrow} X^{(r-1)}
$$

such that $p_{r-1}^{\perp} \circ p_{\perp}^{r}$ is the natural projection

$$
p_{r-1}^{r}: X^{(r)} \longrightarrow X^{(r-1)} .
$$

The fibres of the bundle $X^{(r)} \rightarrow X_{\tau}^{\perp}$ are called principal subspaces in $X^{(r)}$ for the hyperplane distribution $\tau$.

We can now state Gromov's theorem, which gives a large class of differential relations that satisfy the $C^{\perp}$-dense $h$-principle for extensions.

Theorem 5. A differential relation $\mathcal{R} \subset X^{(r)}$ satisfies the $C^{\perp}$-dense $h$-principle for extensions if the following three conditions are satisfied.

(i) The distribution $\tau$ is integrable.

(ii) The subset $\mathcal{R} \subset X^{(r)}$ is open.

(iii) The intersection of $\mathcal{R}$ with any principal space is connected and the convex hull of this intersection is the whole principal space.

This means that given a $C^{r}$-section $f: U \rightarrow X$ and a $C^{0}$-section $\varphi: U \rightarrow \mathcal{R}$ (where $U$ is an open set in $V$ ) such that $J_{f}^{\perp}=p_{\perp}^{r} \circ \varphi$ and $J_{f}^{r}|C=\varphi| C$ for a given closed subset $C$ of $U$, then $\varphi$ can be homotoped to a $C^{\perp}$-close holonomic section over $U$, and the homotopy may be chosen constant near $C$.

Note in particular that the required section $\varphi$ exists if $J_{f}^{r}$ can be homotoped along the fibres of $p_{\perp}^{r}: X^{(r)} \rightarrow X_{\tau}^{\perp}$, that is, along the principal spaces, to a section of $\mathcal{R}$, where the homotopy has to be constant near $C$. Hence, if this purely homotopytheoretic obstruction vanishes, we can find a solution (i.e., a holonomic section) of $\mathcal{R}$.

Although the conditions in Theorem 5 are slightly more complicated than in other cases of Gromov's $h$-principle, the proof [3, pp. 172-173] is relatively short and accessible with little more than a knowledge of the general setup described above. (We have retained Gromov's notation to allow easy reference to this proof.) 
The main idea in the proof of Gromov's theorem is that condition (iii) allows to find a homotopy $\varphi_{s}$ of sections of $\mathcal{R}$ which "surrounds" $J_{f}^{r}$, that is, such that $J_{f}^{r}(u)$ lies in the convex hull of the path of the homotopy $\varphi_{s}(u)$ for all $u \in U$. In this situation one can apply the $C^{\perp}$-approximation lemma [3, pp. 170-171], a special case of Gromov's convex integration technique. The proof of this lemma is independent of earlier parts of Gromov's book. Condition (i) is needed because the proof of Theorem 5 uses a local splitting of $U$ into slices tangent to $\tau$; condition (ii) ensures that small $C^{r}$-perturbations that are used in the approximation process keep us within $\mathcal{R}$.

\section{Proof of Proposition 4}

Let $\alpha_{1}$ be the standard contact form on $S^{3}$ given in Section 2, and define two further contact forms $\alpha_{2}, \alpha_{3}$ on $S^{3} \subset \mathbb{R}^{4}$ by

$$
\begin{aligned}
& \alpha_{2}=x_{1} d x_{3}-x_{3} d x_{1}-x_{2} d x_{4}+x_{4} d x_{2}, \\
& \alpha_{3}=x_{1} d x_{4}-x_{4} d x_{1}+x_{2} d x_{3}-x_{3} d x_{2} .
\end{aligned}
$$

The Reeb vector field $\xi_{1}$ of $\alpha_{1}$ is

$$
\xi_{1}=x_{1} \partial_{x_{2}}-x_{2} \partial_{x_{1}}+x_{3} \partial_{x_{4}}-x_{4} \partial_{x_{3}},
$$

and it is a straightforward check that

$$
\begin{gathered}
\left.L_{\xi_{1}} d \alpha_{2}=d\left(\xi_{1}\right\rfloor d \alpha_{2}\right)=-2 d \alpha_{3}, \\
\left.L_{\xi_{1}} d \alpha_{3}=d\left(\xi_{1}\right\rfloor d \alpha_{3}\right)=2 d \alpha_{2} .
\end{gathered}
$$

Note that the $d \alpha_{i}$ trivialize $\Lambda^{2} T^{*} S^{3}$, so Proposition 4 holds for $M=S^{3}$.

Now, given some 3 -manifold $M$, we use Theorem 3 as was done in [2] to lift $\alpha_{1}$ to a contact form $\omega$ on $M$ (whose Reeb vector field we denote by $\xi$ ) which outside $B \subset M$ is simply the pull-back of $\alpha_{1}$, and which defines a trivial 2-plane bundle $\operatorname{ker} \omega$. Furthermore, we define $\alpha_{0}$ and $\tilde{\alpha}_{0}$ outside $B$ as the pull-back of $\alpha_{2}$ and $\alpha_{3}$, respectively, and extend them as arbitrary 1-forms over $B$. Then $\omega, \alpha_{0}$ and $\tilde{\alpha}_{0}$ are 1-forms on $M$ which satisfy the conditions of Proposition 4 outside $B \subset M$.

Our aim now is to homotope $\alpha_{0}$ and $\tilde{\alpha}_{0}$ to the desired forms $\alpha$ and $\tilde{\alpha}$ by a homotopy which is constant outside a small neighbourhood of $B$. To this extension problem we apply Theorem 5 in the following way. Let $X=T^{*} M \oplus T^{*} M$ and consider the space $X^{(2)}$ of 2 -jets of pairs of 1-forms. Choose a trivialization $\xi^{\prime}, \xi^{\prime \prime}$ of ker $\omega$ which outside $B$ coincides with that given by the Reeb vector fields of $\alpha_{0}$ and $\tilde{\alpha}_{0}$. This choice of $\xi^{\prime}, \xi^{\prime \prime}$ is possible because the bundle $\operatorname{ker} \omega$ is trivial over $B$ and $\pi_{2}\left(S^{1}\right)=0$, so a trivialization given on $\partial B$ extends over $B$. Then the dual 2 -forms $\Omega=d \omega, \Omega^{\prime}, \Omega^{\prime \prime}$ (with respect to $\omega \wedge d \omega$ ) of $\xi, \xi^{\prime}, \xi^{\prime \prime}$ define a trivialization of $\Lambda^{2} T^{*} M$.

Define the relation $\mathcal{R}$ by stipulating that the fibre of $\mathcal{R} \rightarrow M$ over $m$ be given by pairs $\left(j_{m}^{2} \alpha, j_{m}^{2} \tilde{\alpha}\right)$ of 2 -jets of 1 -forms such that $d \omega, L_{\xi} d j_{m}^{2} \alpha, L_{\xi} d j_{m}^{2} \tilde{\alpha}$ are linearly independent and define the same orientation of $\Lambda^{2} T^{*} M$ as $\Omega_{m}, \Omega_{m}^{\prime}, \Omega_{m}^{\prime \prime}$.

Now we have

Lemma 6. The relation $\mathcal{R}$ just defined is open in $X^{(2)}$. Condition (iii) of Theorem 5 is satisfied at points where $\xi$ is transverse to $\tau$. Furthermore, for $U \subset M$ a sufficiently small open set, the projection $\mathcal{R} \rightarrow X^{\perp}$ restricted to $\left(p \circ p_{0}^{2}\right)^{-1}(U)$ is a trivial fibration over $\left(p \circ p_{\perp}^{2}\right)^{-1}(U)$ whose fibres have vanishing second homotopy group $\pi_{2}$. Here $p$ denotes the projection $X=X^{(0)} \rightarrow M$. 
Before giving the proof of this lemma, we complete the proof of Proposition 4. Cover $B$ with a tight cartesian grid, such that in a neighbourhood of every cell of this grid, $\omega$ is in local coordinates given as $\omega=d z-x d y+y d x$ (this is possible by the classical Darboux theorem). In such a neighbourhood, let $\tau$ be the integrable distribution tangent to the foliation $\{z=$ const. $\}$ (This of course does not define a global distribution on $B$ ). Furthermore, we may assume that $\mathcal{R} \rightarrow X^{\perp}$ is a trivial fibration over these neighbourhoods.

If $\alpha$ and $\tilde{\alpha}$ already satisfy the conditions of Proposition 4 near a contractible component of the boundary of such a cell, then Theorem 5 and Lemma 6 allow the extension of $\alpha$ and $\tilde{\alpha}$ over the cell, satisfying the conditions of Proposition 4 everywhere, since in this case there is no homotopy obstruction. Only when we want to extend $\alpha$ and $\tilde{\alpha}$ over the last cell, we meet with a homotopy obstruction in $\pi_{2}(\mathcal{R} \cap$ principal space), which vanishes by Lemma 6 .

In more detail, the vanishing of the homotopy obstructions can be seen as follows. Suppose that over a cell $Q$ we have 1-forms $\alpha$ and $\tilde{\alpha}$ that already satisfy the required independence condition either in a neighbourhood $W$ of a contractible component of the boundary of $Q$ or in a neighbourhood of the whole boundary of $Q$. (In other words, we rule out the case that $\alpha$ and $\tilde{\alpha}$ have to remain fixed during the approximation process near a boundary component that is not simply-connected, since this could lead to a homotopy obstruction.) Our particular choice of a splitting of $B$ into little cells ensures that in the step by step extension over the cells of the grid, these are the only two cases to consider. The jets $j^{2} \alpha$ and $j^{2} \tilde{\alpha}$ give a holonomic section $\sigma$ of $X^{(2)}$ over $Q$, and over $W$ this section lies in $\mathcal{R} \subset X^{(2)}$. Over $Q$, we have chosen a particular distribution $\tau$, which defines the bundle $X_{\tau}^{\perp}$. Gromov's theorem allows to homotope $\alpha$ and $\tilde{\alpha}$ to 1 -forms satisfying the independence condition of Proposition 4 everywhere over $Q$ if we can find a homotopy of $\sigma$ along the principal spaces, i.e., the fibres of $X^{(2)} \rightarrow X_{\tau}^{\perp}$, to a (non-holonomic) section $\varphi$ of $\mathcal{R} \rightarrow Q$. Since the fibres of $X^{(2)} \rightarrow X_{\tau}^{\perp}$ are affine spaces, it is enough to find a section $\varphi$ of $\mathcal{R} \rightarrow Q$ such that $\varphi(m)$ lies in the same principal space as $\sigma(m)$. Let $\sigma^{\perp}=p_{\perp}^{2} \circ \sigma$ be the section of $X_{\tau}^{\perp}$ over $Q$ induced by $\sigma$. Then it is an equivalent problem to find a section of the bundle $\left(\sigma^{\perp}\right)^{*} \mathcal{R}$ over $Q$, where the section has to take prescribed values in a neighbourhood $W^{\prime} \subset W$ of the respective boundary component of $Q$. Since the bundle $\left(\sigma^{\perp}\right)^{*} \mathcal{R}$ over $Q$ is a trivial bundle whose fibre has vanishing second homotopy group, this extension problem can always be solved.

It remains to prove Lemma 6 . That $\mathcal{R}$ is open is clear. The remaining statements are purely local in character, so we fix a point $m$ in $M$ and consider a pair of 2jets of 1 -forms $\left(j_{m}^{2} \alpha, j_{m}^{2} \tilde{\alpha}\right)$ at $m$. In the sequel, we suppress the index $m$. The transversality assumption on $\xi$ and $\tau$ allows to assume that in local coordinates near $m$ we have $\xi=\partial_{z}$ and $\tau$ is the distribution tangent to $\{z=$ const. $\}$. The 2 -jet $j^{2} \alpha$ can be represented as

$$
\begin{aligned}
j^{2} \alpha= & (x, y, z) R\left(\begin{array}{l}
x \\
y \\
z
\end{array}\right) d x+(x, y, z) S\left(\begin{array}{l}
x \\
y \\
z
\end{array}\right) d y+(x, y, z) T\left(\begin{array}{l}
x \\
y \\
z
\end{array}\right) d z \\
& +(x, y, z) A\left(\begin{array}{l}
d x \\
d y \\
d z
\end{array}\right)+\left(a_{1}, a_{2}, a_{3}\right)\left(\begin{array}{l}
d x \\
d y \\
d z
\end{array}\right),
\end{aligned}
$$

where $R=\left(R_{i j}\right), S=\left(S_{i j}\right)$ and $T=\left(T_{i j}\right)$ are symmetric $(3 \times 3)$-matrices with constant entries, $A$ is a constant $(3 \times 3)$-matrix, and the $a_{i}$ are constant real numbers. 
Note that in a fixed principal space, the only free variables in this expression are $R_{33}, S_{33}$ and $T_{33}$.

A straightforward calculation shows that $L_{\xi} d j^{2} \alpha$ is given by

$$
\begin{aligned}
L_{\xi} d j^{2} \alpha= & 2\left(T_{13}-R_{33}\right) d x \wedge d z+2\left(T_{23}-S_{33}\right) d y \wedge d z \\
& + \text { multiples of } d x \wedge d y .
\end{aligned}
$$

Similarly,

$$
\begin{aligned}
L_{\xi} d j^{2} \tilde{\alpha}= & 2\left(\widetilde{T}_{13}-\widetilde{R}_{33}\right) d x \wedge d z+2\left(\widetilde{T}_{23}-\widetilde{S}_{33}\right) d y \wedge d z \\
& + \text { multiples of } d x \wedge d y .
\end{aligned}
$$

The condition that $L_{\xi} d j^{2} \alpha, L_{\xi} d j^{2} \tilde{\alpha}$ and $d \omega_{m}=d x \wedge d y$ be linearly independent and define a preferred orientation, translates in the intersection $\mathcal{R}_{x}$ of $\mathcal{R}$ with a principal space $\left(p_{\perp}^{2}\right)^{-1}(x), x \in X^{\perp}$, into a condition on the free variables $R_{33}, S_{33}$, $T_{33}, \widetilde{R}_{33}, \widetilde{S}_{33}, \widetilde{T}_{33}$ as follows. The $T$-components are free, so we get a factor $\mathbb{R}^{2}$; the linear independence and orientation condition becomes

$$
\left(T_{13}-R_{33}\right)\left(\widetilde{T}_{23}-\widetilde{S}_{33}\right)-\left(T_{23}-S_{33}\right)\left(\widetilde{T}_{13}-\widetilde{R}_{33}\right)>0 .
$$

Changing affine coordinates to $x_{1}, x_{2}, x_{3}, x_{4}$ with

$$
\begin{aligned}
& T_{13}-R_{33}=x_{1}+x_{3}, \\
& T_{23}-S_{33}=x_{2}+x_{4}, \\
& \widetilde{T}_{13}-\widetilde{R}_{33}=x_{4}-x_{2}, \\
& \widetilde{T}_{23}-\widetilde{S}_{33}=x_{1}-x_{3},
\end{aligned}
$$

we obtain the affine cone $\left\{x_{1}^{2}+x_{2}^{2}-x_{3}^{2}-x_{4}^{2}>0\right\}$.

Hence

$$
\mathcal{R}_{x} \cong \mathbb{R}^{2} \times\left\{x_{1}^{2}+x_{2}^{2}-x_{3}^{2}-x_{4}^{2}>0\right\} \subset \mathbb{R}^{6} \cong\left(p_{\perp}^{2}\right)^{-1}(x) .
$$

It is now easy to see that the convex hull of $\mathcal{R}_{x}$ in $\left(p_{\perp}^{2}\right)^{-1}(x)$ is all of $\left(p_{\perp}^{2}\right)^{-1}(x)$ and that $\mathcal{R}_{x}$ is homeomorphic to $S^{1} \times \mathbb{R}^{5}$. In particular, $\pi_{2}\left(\mathcal{R}_{x}\right)=\pi_{2}\left(S^{1}\right)=0$. The explicit coordinate description of the $\mathcal{R}_{x}$ also implies the statement about the local triviality of the projection $\mathcal{R} \rightarrow X^{\perp}$.

This completes the proof of Lemma 6 .

Next we give an outline of the proof of Theorem 2. The method of proof is essentially the same as that for Theorem 1 . As before, we first construct a contact form $\omega_{1}$ on $M$ with trivial 2-plane bundle $\operatorname{ker} \omega_{1}$. This allows to find 1-forms $\alpha_{0}, \tilde{\alpha}_{0}$ on $M$ such that $\omega_{1} \wedge \alpha_{0} \wedge \tilde{\alpha}_{0}$ is nowhere zero. Furthermore, as in the proof of Proposition 4, we may assume that $d \omega_{1}, d \alpha_{0}, d \tilde{\alpha}_{0}$ are linearly independent everywhere outside a 3 -ball $B \subset M$.

Now let $X=T^{*} M \oplus T^{*} M$ and consider the relation $\mathcal{R} \subset X^{(1)}$ given by pairs of 1-jets of 1 -forms $\left(j_{m}^{1} \alpha, j_{m}^{1} \tilde{\alpha}\right)$ such that $d \omega_{1, m}, d j_{m}^{1} \alpha, d j_{m}^{1} \tilde{\alpha}$ are linearly independent and define a preferred orientation. This $\mathcal{R}$ also satisfies Lemma 6 (in fact, in suitable affine coordinates, $\mathcal{R}_{x} \subset\left(p_{\perp}^{1}\right)^{-1}(x)$ looks exactly the same as for the relation we considered in Lemma 6), so we find 1-forms $\alpha$ and $\tilde{\alpha}$ on $M$ such that $d \omega, d \alpha, d \tilde{\alpha}$ are everywhere linearly independent.

Since we are using a $C^{\perp}$-approximation of 1-jets, $\alpha$ and $\tilde{\alpha}$ can be chosen $C^{0}$-close to $\alpha_{0}$ and $\tilde{\alpha}_{0}$, respectively. This implies that we can still satisfy the independence condition $\omega_{1} \wedge \alpha \wedge \tilde{\alpha} \neq 0$. Then $\omega_{1}, \omega_{2}=\omega_{1}+\epsilon \alpha$ and $\omega_{3}=\omega_{1}+\epsilon \tilde{\alpha}$ are the desired contact forms for $\epsilon>0$ a sufficiently small constant. 
Remark. Strictly speaking, Theorem 3 is not essential for the proof of Theorems 1 and 2, and we shall see below how Theorem 2 generalizes to higher dimensions. However, the use of Theorem 3 greatly simplifies the argument in two places.

First of all, as shown in [2], it allows the direct construction of a contact form $\omega_{1}$ with trivial 2-plane bundle ker $\omega_{1}$. Note that the $h$-principle fails for the extension of contact forms, so one does need some geometric structure theorem for the construction of a suitable $\omega_{1}$. In higher dimensions, we do not have such a geometric structure theorem, so we shall have to assume that we are given a contact form whose contact distribution admits a certain number of linearly independent sections.

Secondly, Theorem 3 allows to reduce the construction of two further contact forms $\omega_{2}, \omega_{3}$ to a step by step extension problem to which the $h$-principle applies and where all homotopy-theoretic obstructions vanish for a simple geometric reason. Without this geometric information, one needs a more careful analysis of the geometry in the overlap of regions where we use different local distributions $\tau$, to ensure that one does not create any homotopy-theoretic obstructions. Observe that in the proof of Proposition 4, due to the special geometry of the problem, we were free to choose a non-holonomic section $\varphi$ of $\mathcal{R}$ at every step of the extension, as long as we satisfied $p_{\perp}^{2} \circ \varphi=p_{\perp}^{2} \circ \sigma$, where $\sigma$ was the given holonomic section of $X^{(2)}$. (Note that this condition on $\varphi$ determines $p_{0}^{2} \circ \varphi=p_{0}^{2} \circ \sigma$; this is essential to obtain a $C^{0}$-close approximation.) In the general setting, we shall have to start with a global non-holonomic section $\varphi$ of $\mathcal{R}$ and ensure that we still have a global section after every step of the extension.

\section{A Generalization to Higher Dimensions}

Theorem 2 admits the following generalization.

Theorem 7. Let $M$ be a closed, orientable $(2 n+1)$-dimensional manifold with contact form $\omega$, and suppose that the contact distribution $\operatorname{ker} \omega$ admits $k$ everywhere linearly independent sections. Then $M$ admits $(k+1)$ everywhere linearly independent contact forms with everywhere linearly independent Reeb vector fields.

Proof. Analogous to the proof of Theorem 2, the contact forms $\omega_{0}, \ldots, \omega_{k}$ are constructed as $\omega_{0}=\omega, \omega_{i}=\omega+\epsilon \alpha_{i}$ for $i=1, \ldots, k$, where the $\alpha_{i}$ are 1 -forms such that

$$
(d \omega)^{n},(d \omega)^{n-1} \wedge d \alpha_{1}, \ldots,(d \omega)^{n-1} \wedge d \alpha_{k}
$$

are everywhere linearly independent. This condition implies that (for $\epsilon>0$ sufficiently small) the $\omega_{i}$ are contact forms with everywhere linearly independent Reeb vector fields. If the $\alpha_{i}$ can be found $C^{0}$-close to $\alpha_{i}^{0}$, where $\omega, \alpha_{1}^{0}, \ldots, \alpha_{k}^{0}$ are everywhere linearly independent 1 -forms, then $\omega_{0}, \ldots, \omega_{k}$ will be everywhere linearly independent.

Let $X$ be the Whitney sum of $k$ copies of $T^{*} M$, and define the relation $\mathcal{R} \subset X^{(1)}$ as before by requiring the linear independence of the differentials of the $k 1$-jets of 1 -forms (together with $d \omega_{m}$ ). If $k=2 n$, we also fix an orientation to make $\mathcal{R}$ connected. A similar calculation as in the proof of Lemma 6 shows that $\mathcal{R}$ satisfies the conditions of Gromov's theorem.

Let $Y_{1}, \ldots, Y_{k}$ be everywhere linearly independent sections of ker $\omega$, which exist by the assumptions of the theorem. Set

$$
\left.\alpha_{i}^{0}=Y_{i}\right\rfloor d \omega
$$


and

$$
\Omega_{i}=\omega \wedge \alpha_{i}^{0}
$$

Then the $\left(\alpha_{i}^{0}, \Omega_{i}\right)$ define a global smooth, non-holonomic section $\varphi$ of $\mathcal{R}$. The 1-jets of the $\alpha_{i}^{0}$ define a global holonomic section $\sigma=J^{1}\left(p_{0}^{1} \varphi\right)$ of $X^{(1)}$.

Cover $M$ with finitely many open balls $B_{\nu}$ such that in each ball, $\omega$ can be written in local coordinates as $\omega=d z-\sum_{i=1}^{n}\left(x_{i} d y_{i}-y_{i} d x_{i}\right)$, and such that $\mathcal{R} \rightarrow X^{\perp}$ is a trivial fibration over each $B_{\nu}$. We call such a cover $\left\{B_{\nu}\right\}$ a good cover. The distribution $\tau$ is defined locally as in the proof of Proposition 4.

Now we have the following lemma.

Lemma 8. Let $\varphi$ be a global smooth, non-holonomic section of $\mathcal{R}$ and let $\left\{B_{\nu}\right\}$ be a good cover of $M$. Suppose that in the intersection $W$ of a fixed ball $B=B_{\nu_{0}}$ with certain other balls $B_{\nu_{i}}$, the section $\varphi$ is already holonomic. Then one can find a global section $\tilde{\varphi}$ of $\mathcal{R}$ that satisfies the following conditions:

(i) $p_{0}^{1} \tilde{\varphi}$ is $C^{0}$-close to $p_{0}^{1} \varphi$,

(ii) $\tilde{\varphi}=\varphi$ outside $B$,

(iii) $\tilde{\varphi}=\varphi$ near the intersection $\widetilde{W} \subset W$ of $B$ with slightly smaller balls $\widetilde{B}_{\nu_{i}} \subset$ $B_{\nu_{i}}$

(iv) If $W$ covers the $2 n$-sphere $\partial B=\bar{B}-B, \tilde{\varphi}$ is holonomic everywhere over $B$; if $W$ does not cover $\partial B, \tilde{\varphi}$ is only required to be holonomic in a slightly smaller ball $\widetilde{B}$.

Furthermore, the $\widetilde{B}_{\nu_{i}}$ and $\widetilde{B}$ may be chosen arbitrarily close to $B_{\nu_{i}}$ and $B$, respectively, so that these slightly smaller balls, together with the $B_{\nu}$ not involved in the construction, still form a good cover of $M$.

It is clear that Theorem 7 follows by repeated application of this extension lemma.

Proof of Lemma 8. Consider the sections $\varphi^{\perp}=p_{\perp}^{1} \varphi$ and $\sigma^{\perp}=p_{\perp}^{1} \sigma$ (where $\sigma=$ $\left.J^{1}\left(p_{0}^{1} \varphi\right)\right)$ of $X^{\perp}$ over $B$. Since $X^{\perp} \rightarrow B$ is an affine bundle, $\varphi^{\perp}$ and $\sigma^{\perp}$ are homotopic by a linear homotopy $\varphi_{t}^{\perp}$, and this homotopy is constant over $W$. The section $\varphi^{\perp}$ lifts to the section $\varphi$ of $\mathcal{R}$, and since $\mathcal{R} \rightarrow X^{\perp}$ is a trivial fibration over $B$, the homotopy $\varphi_{t}^{\perp}$ lifts to a homotopy $\varphi_{t}$ which is constant over $W$, i.e., we have

(a) $\varphi_{t}: B \rightarrow \mathcal{R}, 0 \leq t \leq 1$,

(b) $\varphi_{0}=\varphi$,

(c) $p_{\perp}^{1} \varphi_{1}=\sigma^{\perp}$,

(d) $\varphi_{t}|W=\varphi| W$.

The condition $p_{\perp}^{1} \varphi_{1}=\sigma^{\perp}$ means that for every $m \in B$, both $\sigma(m)$ and $\varphi_{1}(m)$ lie in the same principal space. Then Gromov's theorem allows to homotope $\varphi_{1}$ to a holonomic section $\sigma_{1}$ that is $C^{\perp}$-close to $\sigma$ and such that the homotopy is constant near $\widetilde{W}$, where $\widetilde{W}$ is as defined in condition (iii) of the lemma. Gromov's theorem allows to choose the $\widetilde{B}_{\nu_{i}}$ as close to the $B_{\nu_{i}}$ as we wish.

If $W$ covers $\partial B$ (in which case we may assume that $\widetilde{W}$ also covers $\partial B$, the last statement of the lemma therefore holds), set $\tilde{\varphi}=\sigma_{1}$ in $B$ and $\tilde{\varphi}=\varphi$ outside $B$.

If $W$ does not cover $\partial B$, choose two slightly smaller balls $\widetilde{B} \subset B^{\prime} \subset B$. Then set $\tilde{\varphi}=\sigma_{1}$ in $\widetilde{B}$, in the annulus between $\partial \widetilde{B}$ and $\partial B^{\prime}$ we define $\tilde{\varphi}$ by the homotopy from $\sigma_{1}$ to $\varphi_{1}$, in the annulus between $\partial B^{\prime}$ and $\partial B$ we set $\tilde{\varphi}=\varphi_{\chi(t)}$ (where $\chi(t) \equiv 1$ near $\partial B^{\prime}$ and $\chi(t) \equiv 0$ near $\partial B$ ), and outside $B$ we set $\tilde{\varphi}=\varphi$. Again, $\widetilde{B}$ may be 
chosen as close to $B$ as we like, so the slightly smaller balls will still form a good cover.

It remains to check conditions (i)-(iv). It is obvious from the construction that (ii) and (iv) are satisfied. Both homotopies involved in the construction are constant near $\widetilde{W}$; this implies (iii). From Gromov's theorem we know that $p_{0}^{1} \varphi_{1}$ and $p_{0}^{1} \sigma_{1}$ are $C^{0}$-close and so is the homotopy between them. Since $\varphi_{t}$ is the lift of the linear homotopy $\varphi_{t}^{\perp}$ in the fibres of $X^{\perp} \rightarrow B$, we clearly have $p_{0}^{1} \varphi_{t}=p_{0}^{1} \varphi$. This proves (i) and completes the proof of Lemma 8.

\section{REFERENCES}

[1] H. Geiges and C.B. Thomas, Hypercontact manifolds, J. London Math. Soc. (2) 51 (1995), 342-352. CMP 95:10

[2] J. Gonzalo, Branched covers and contact structures, Proc. Amer. Math. Soc. 101 (1987), 347-352. MR 88k:53058

[3] M. Gromov, Partial Differential Relations, Springer-Verlag, Berlin, New York, 1986. MR 90a:58201

[4] H.M. Hilden, J.M. Montesinos, and T. Thickstun, Closed oriented 3-manifolds as 3-fold branched coverings of $S^{3}$ of spherical type, Pacific J. Math. 65 (1976), 65-76. MR 54:8635

Department of Mathematics, Stanford University, Stanford, California 94305-2125

Current address: Departement Mathematik, ETH Zentrum, 8092 Zürich, Switzerland

E-mail address: geiges@math.ethz.ch

Departamento de Matemáticas, Universidad Autónoma de Madrid, 28049 Madrid, SPAIN

E-mail address: jgonzalo@ccuam3.sdi.uam.es 